\title{
Parametric analysis of magnetic field mitigation shielding for underground power cables
}

\author{
J. C. del Pino López ${ }^{1}$ P. Cruz Romero ${ }^{1}$ and P. Dular ${ }^{2}$ \\ ${ }^{1}$ Department of Electrical Engineering, University of Seville \\ Camino de los Descubrimientos, s/n - 41092 Sevilla, Spain \\ phone: +34 954481277 - fax: +34 954487284 - e-mail: plcruz@us.es \\ ${ }^{2}$ Department of Electrical Engineering and Computer Science, University of Liège \\ Sart-Tilman, Bldg. B28, Parking P32 B-4000 Liège, Belgium \\ phone: +32 43663710 - e-mail: patrick.dular@ulg.ac.be
}

\begin{abstract}
In this paper, an exhaustive analysis of the most important parameters that affect to the magnetic field mitigation efficiency of the shield is done, in order to obtain the best shield configuration depending on the source caracteristics, region to be shielded and the mitigation requirements. This is done using a free software based on the finite elements method.
\end{abstract}

\section{Key words}

Magnetic field, shielding, eddy currents, underground power cables, finite elements method.

\section{Introduction}

Possible adverse health effects due to power frequency magnetic fields (PFMFs) have become one of the most discussed issues related to public health in the last two decades. Numerous epidemiological and laboratory studies have been done. Unfortunately the main conclusion is that there is no clear evidence of adverse health effects at the levels of PFMFs to which people are normally exposed. However, from the studies carried out until now can not be concluded firmly that there is no link between PFMFs and some certain diseases. The lack of certainty has driven main national and international health and radiological organizations to recommend prudent avoidance, i.e., taking precautionary measurements to avoid the long-term exposition to high fields (precautionary principle) [1]. Also, some countries have developed new regulations involving a drastic reduction of the allowable limits of exposure, both for new constructions as well as for existing systems that must be reconfigured. Due to these reasons, remarkable efforts have been devoted to research activities aiming at studying suitable techniques to achive the desired reduction of magnetic fields generated by electrical systems. In many cases this can be done by simple and effective solutions that do not mean a costly additional investment. However, in other types of PFMFs sources, managing these fields is still a challenge to researchers. One of these PFMFs sources are the high voltage transmission underground cables. Several techniques have been applied in these sources to mitigate the magnetic field that can be classified in two goups: "intrinsic methods", when they act on the laying parameters to reduce the field strength, like phase arrangement, and "external shielding techniques", when special apparatuses external to the cable are used, such as grids of insulated conductors and shields made of ferromagnetic or conductive materials $[2,3,4]$. These techniques have proved to be efficient, reducing the field at a great percentage in some cases. All the techniques have advantages and drawbacks. So, a site-specific information should be provided in order to decide the best solution.

It is clear that, in order to obtain the best shield configuration depending on the source caracteristics, region to be shielded and the mitigation requirements, an exhaustive analysis of the most important parameters that affect to the mitigation efficiency of the shield is needed. In this paper, the influence of those parameters have been analyzed in several shielding configurations. For that purpose, a free software based in the finite elements method (FEM) has been used, obtaining important data and graphical results of the influence of those parameters in the magnetic field and the reduction factor in the proximities of the field source.

\section{Magnetic field shielding}

Magnetic field management is basically concerned with the minimization of the effect of magnetic fields on the public health without sacrificing the effectiveness and reliability of the power system. There are a number of design options that would reduce the magnetic field in a certain area. Most of them can be classified in two main categories: (a) shielding a subject and (b) shielding a source. Shielding a subject consists in the implementation of some kind of shield in order to reduce the field in some relatively small volume, due to sources of field outside the volume (Fig. 1a). This technique is mainly used in places where high 
accuracy equipments are used, as, for example, some kind of laboratories.

On the other hand, shielding the source involves placing a shield in the proximities or surrounding some localized field source to reduce the magnetic field in the "outside" world from the source (Fig. 1b). This is the case of underground power lines.

a)

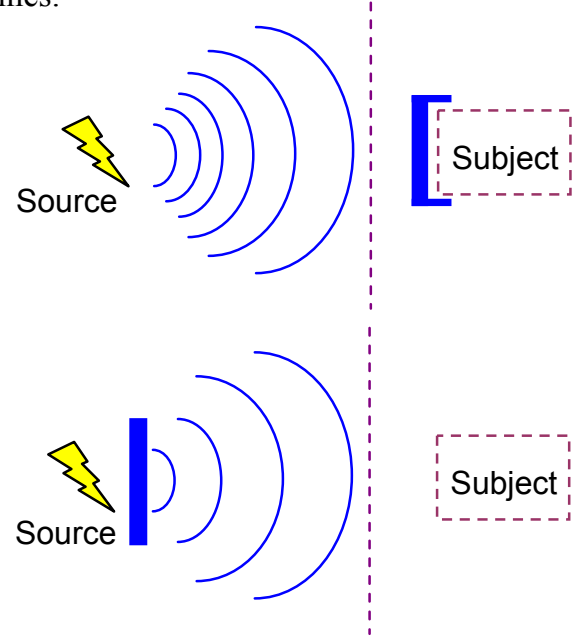

Fig. 1. a) Shielding a subject, b) shielding a source.

In any of these cases, the field is reduced by establishing currents in a shield made with conducting materials in such a way that fields produced by those currents oppose the field to be reduced. Theses currents can be established in two ways: they can be imposed by external device (active shielding) or can be induced by the source to be shielded (passive shielding). This is the case studied in this paper.

\section{Shielding of underground power cables}

In order to mitigate the magnetic field caused by underground power cables, one of the most efficient solutions is the shielding technique. It can be done in two different shield topologies: open shields and close shields. The first type consists on a flat plate located above the source (Fig. 2a), and the second one surrounds the field source (Fig. 2b). The latter one is generally used in new constructions and is more expensive than the first one, which is usually employed to reconfigure existing systems.

In any case, both of them are usually made of highly conductive materials (aluminium, copper) or ferromagnetic materials (iron, low carbon steel, $\mu$-metal). This aspect is important, because each material provides a different shielding method. If high conductive materials are used, the mitigation of the field is based on a mechanism known as "eddy current cancellation". In this case, currents are induced in the conductor, which cause magnetic fields that partially cancel those of the source (Fig. 3).

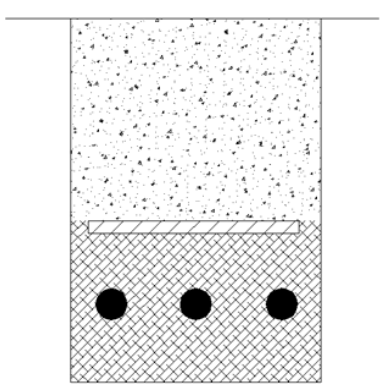

a)

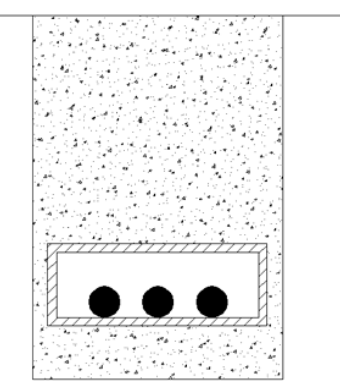

b)
Fig. 2. Open shield (a) and close shield (b).

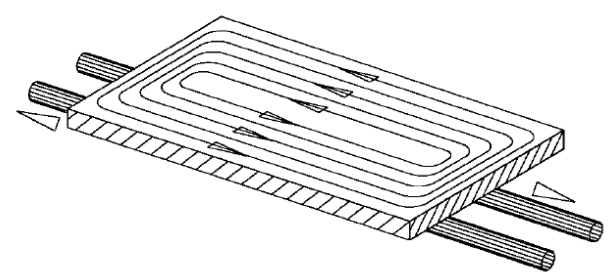

Fig. 3. Eddy currents induced in a metal plate.

If high permeability material is used, the mechanism is known as "flux shunting". In this case, the magnetic flux from the source is diverted into the magnetic material and away from the region to be shielded (Fig. 4). These two shielding methods are characterized by different boundary conditions which have to be considered to analyze each configuration.

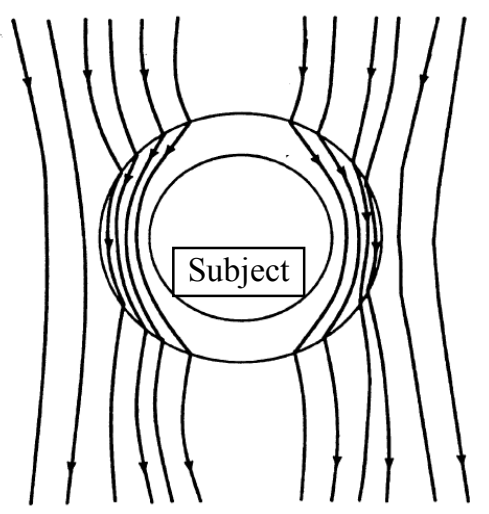

Fig. 4. "Flux shunting" mechanism.

It is not trivial to decide which shielding method is the best to reduce the field caused by an underground power line, because the shielding effectiveness depends on many kinds of parameters, such as the configuration of the line (flat, trefoil), width and thickness of the plate, mutual distance between shield and cables, etc. Another aspect to consider is the fact that the results obtained with conductive materials differ from those obtained using magnetic materials. The last one usually provides high field reduction in the proximities of the source, and the first one gives 
better results far from the source. This is also strongly affected by the laying configuration of the power cables (horizontal, vertical, trefoil). These are some of the reasons why the use of high conductive open shields is suitable in case of flat lines, whereas in case of vertical lines shields made of ferromagnetic materials are better performing [4]. On the other hand, it is also useful to consider that magnetic materials are more expensive than conductive materials.

Due to all these reasons, an exhaustive analysis of these parameters that influence the shielding effectiveness is done, in order to facilitate the selection of the configuration best suited for a particular system. The parameters analyzed are:

- Thickness of the plate.

- Distance from the source and relationship with dimensional characteristics of the source.

- $\quad$ Profile of the plate.

- Magnetic permeability value.

- Using different types of materials.

\section{Analysis}

Unfortunately, there is not currently a simple method to analyze this kind of problems. In the last years, numerical methods have been proved to be a suitable tool to use in magnetic shielding analysis, but some approximations have to be used before starting the analysis. If it is assumed that the field generated by the underground line only has two components (the third can be neglected if the field is calculated at several meters far from both ends of the line, and with a straight stretch) the analysis is $2-\mathrm{D}$, so the geometrical configuration (Fig. 2) and the field generated can be represented in a plane (Fig. 4). Taking this into account, 2-D analysis and calculations have been carried out using free numerical simulation software based on finite elements method, developed by University of Liege (http://geuz.org/getdp/). This software provides data and diagrams resulting from numerical solutions of the different configurations analyzed.

In particular, three different shielding configurations have been studied based on a flat arrangement of the underground power line: horizontal plate shield, reverse-U shield and closed shield surrounding the three conductors using conductive and ferromagnetic materials. In all the cases, the three conductors are supplied by balanced currents of $100 \mathrm{~A}$ at the frequency of $50 \mathrm{~Hz}$ (the effect of the current is not important on the results). Cables depth is fixed at $80 \mathrm{~cm}$, with a separation between cables of $25 \mathrm{~cm}$ and conductors diameters of $20 \mathrm{~mm}$. All data are suitable for voltages about $132 \mathrm{kV}$.

Several analysis have been developed studying the influence of geometrical parameters of the line and the shield in the field created at a $1 \mathrm{~m}$ height above the ground. Moreover, influence in the reduction factor (also called shielding factor) will be considered. It is defined as:

$$
R F=\frac{B_{o}}{B}
$$

where $B_{o}$ is the rms value of the magnetic field without the shield, and $B$ is the rms value of the field with the shield in place. Clearly, $R F$ is a function of position. The biggest $R F$ is, the more significant the magnetic field reduction.

Four different conductive and ferromagnetic materials have been considered in this study: aluminum, steel, $80 \% \mathrm{Ni}-\mathrm{Fe}$ $\mu$ metal and Fair Rite - Type 76 ferrite, with conductivity $(\sigma)$ and relative permeability $\left(\mu_{\mathrm{r}}\right)$ shown in Table I.

TABLE I. - Properties of shielding materials

\begin{tabular}{|c|c|c|}
\hline Material & $\begin{array}{c}\text { Conductivity } \\
(\sigma) \mathrm{S} / \mathrm{m}\end{array}$ & $\begin{array}{c}\text { Relative } \\
\text { Permeability }\left(\mu_{\mathrm{r}}\right)\end{array}$ \\
\hline Aluminum & $3.8 \times 10^{7}$ & 1 \\
\hline Steel & $10^{7}$ & 500 \\
\hline $80 \% \mathrm{Ni}-\mathrm{Fe} \mu$ metal & $1.64 \times 10^{6}$ & 15,120 \\
\hline $\begin{array}{c}\text { Fair Rite - Type } 76 \\
\text { Ferrite }\end{array}$ & 2 & 10,000 \\
\hline
\end{tabular}

\section{A. Horizontal plate shield}

When an underground power line has to be shielded, the most common and cheaper solution is to place a horizontal plate of any conductive or ferromagnetic material above the cables (Fig. 2a). Usually, aluminum plates are employed because of their low cost. This is a high conductivity material in which eddy currents are induced, resulting a high reduction in the magnetic field in the area of interest ( $1 \mathrm{~m}$ above ground). Other materials can be used, producing different levels of field reduction. Fig. 5 shows the lateral profiles of the magnetic field resulting from the use aluminum, steel and ferrite material in the shield.

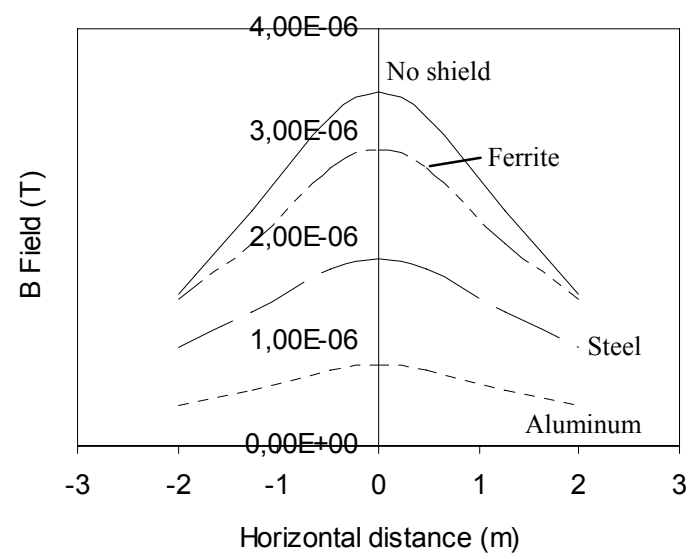

Fig. 5. Lateral profile of B with different shielding materials. 
As can be seen, the most important reduction of the field in the area of interest is achieved by the aluminum shield, followed by the steel shield. The ferromagnetic material has poor results in this case. This suggests that high conductive materials are best suited for reducing the field in areas far from the field source. This can be explained by the reduction mechanism used in each material. Fig. 6a shows how an aluminum shield deforms field lines weaking them near the shield, while ferrite material (Fig. 6b) can only divert field lines in the proximities of the shield into the plate.

a)

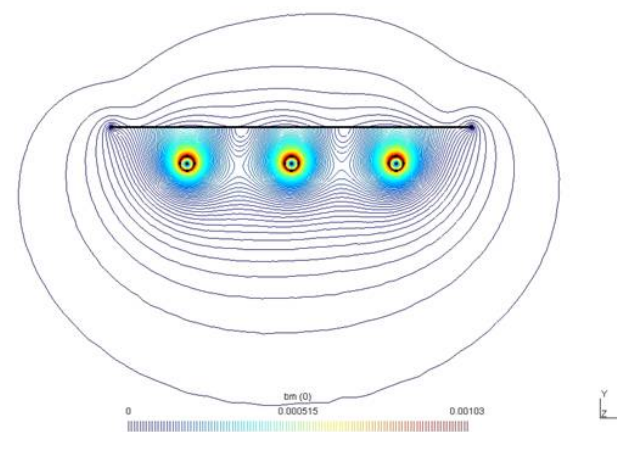

b)

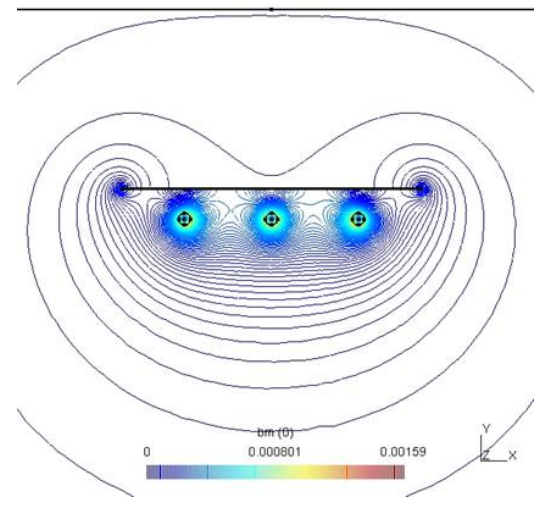

Fig. 6. Field lines in plate shield of Aluminum (a) and Ferrite (b).

The lateral profiles also show that the maximum of the magnetic field at $1 \mathrm{~m}$ height is produced in the centre of the power line, so, in the next analysis, influence in the reduction factor in that point will be discussed.

An important parameter that has high influence in the final cost of the shield is the plate thickness. There have been considered values from 1 to $10 \mathrm{~mm}$ in aluminum, steel and ferrite shields. Evolution of the reduction factor with the plate thickness is shown in Fig. 7. It increases noticeably for thickness varying from 1 to $5 \mathrm{~mm}$, when an aluminum shield is employed. For higher values of the thickness the RF increases slowly. Such trend suggests that it is convenient to use aluminum shields with thickness smaller than $\delta / 2$, being $\delta=13.45 \mathrm{~mm}$ the skin depth of aluminum at $50 \mathrm{~Hz}$. In general the value of $3 \mathrm{~mm}$ is recommended.
On the other hand, thickness influence in steel and ferrite materials can be neglected. Only steel has a remarkable variation in RF at thickness from $1 \mathrm{~mm}$ to $3 \mathrm{~mm}$. So this suggests that any plate thickness using these materials has the same field reduction in the point of study.

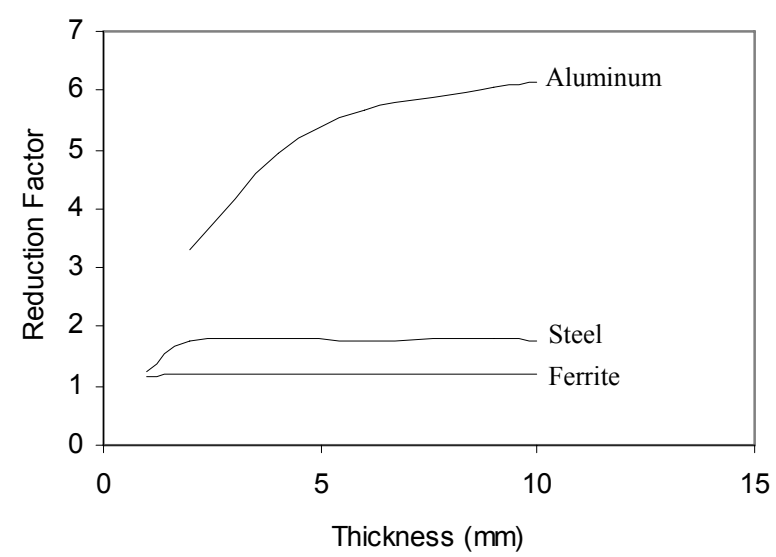

Fig. 7. Reduction factor evolution with plate thickness.

Another important geometric parameter of the shield is the width of the plate. Independently of the material employed in the shield, it's easy to conclude that the larger the width is, the better the reduction obtained (Fig. 8). If a high conductive material is used, more surface implies larger eddy currents path and higher the oppose field will be. Also, if a ferromagnetic material is used, with a larger width, more field lines will be "shunted" into the shield and the resulting field will be lower.

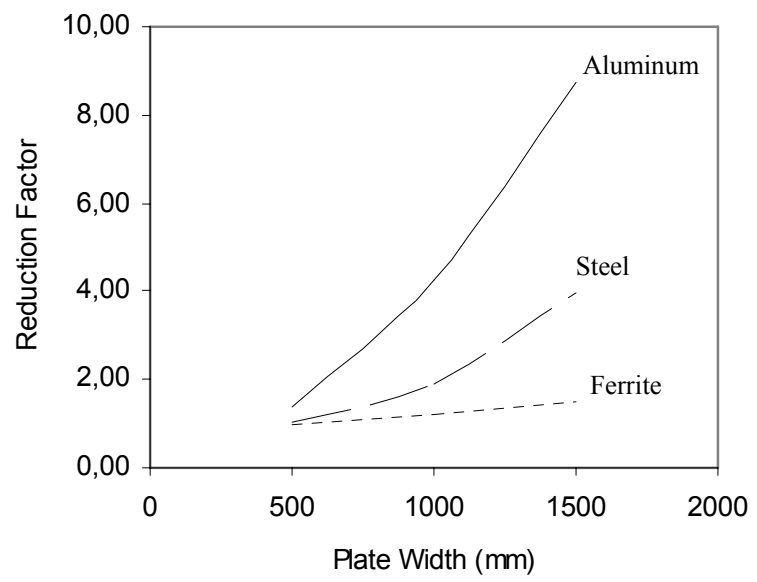

Fig. 8. Influence of the plate width on the reduction factor.

As trench width is usually about $1 \mathrm{~m}$, a reasonable value to consider is from $800 \mathrm{~mm}$ to $1000 \mathrm{~mm}$ of shield width. Anyway, if a larger shield can be placed, better results will be obtained.

Other parameters that have effect in the reduction factor are geometrical parameters of the power cables and the situation of the shield from the source. In this matter, 
several simulations have been developed taking different values of mutual distance between the plate and the line, and separation among power cables. Fig. 9 shows the evolution of RF with the distance between the shield and the cables, varying from $0.5 \mathrm{~mm}$ to $450 \mathrm{~mm}$ of separation. It is clear that, the closer the shield is, the best the reduction factor obtained. This behaviour is shown in both conductive and ferromagnetic materials, since each material employs a different shielding method that improves in short distances, but in latter ones the effect is quite low.

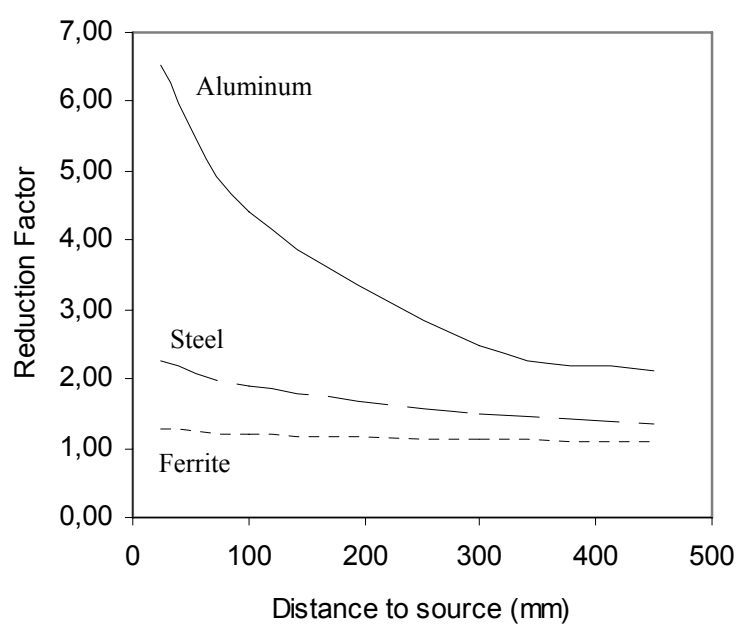

Fig. 9. Distance between plate and power cables.

Taking this into account, values from $50 \mathrm{~mm}$ to $200 \mathrm{~mm}$ are recommended, but it's required analyzing the effect of heat losses in the ampacity of the power line in the particular system. This also has to be analyzed if mutual distance between cables is modified. Fig. 10 shows the evolution of the RF in this case.

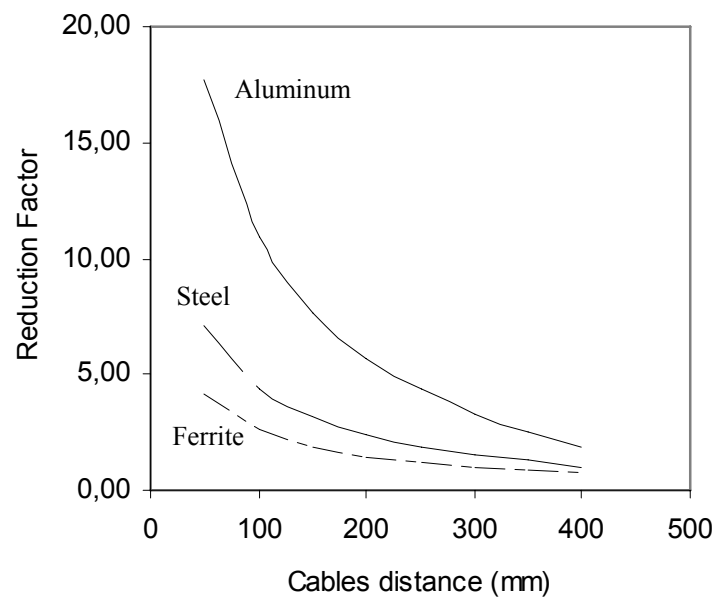

Fig. 10. Distance between cables.

As can be seen, the best solution would be the three conductors in contact or even in trefoil configuration, as it's well known.

\section{B. Reverse-U shield}

If it is possible, another shielding solution is to use a reverse-U shape shield (Fig. 13). Simulations with this kind of shield have been done, employing materials listed in Table I. Fig. 11 shows lateral profile of the field obtained at $1 \mathrm{~m}$ height with each material. It can be seen that better field reduction than horizontal plate is achieved.

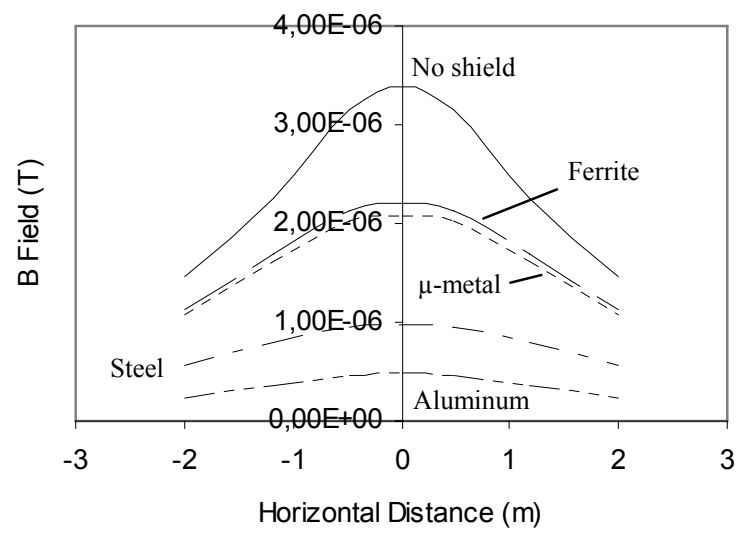

Fig. 11. Lateral profile of B with different shielding materials.

As in the previous case was mentioned, conductive materials have a better behaviour in areas far from the field source, while ferromagnetic material has a better performance in the proximities of the cables. This is supported by Fig 12 where evolution of RF in a cross section in the vertical axis is shown.

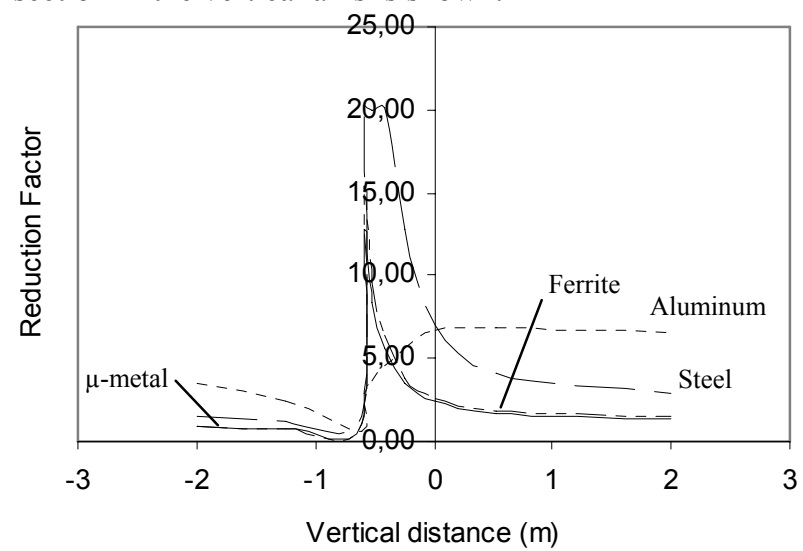

Fig. 12. RF in vertical distance.

It can be seen how ferromagnetic materials have a higher RF value than conductive materials near the cables, but decreases rapidly having at the interest point $(1 \mathrm{~m})$ a lower $\mathrm{RF}$ value than aluminum or steel.

Fig. 13 shows the influence of the plate shape in the field lines. It is clear that the added lateral plates improve the shielding efficiency with all the materials employed, obtaining better results than the horizontal plate case. 
a)

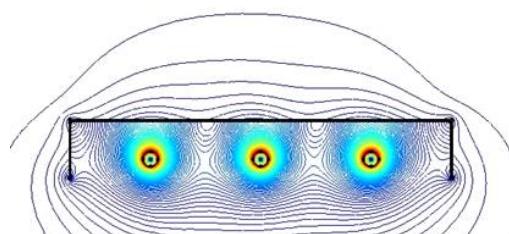

b)

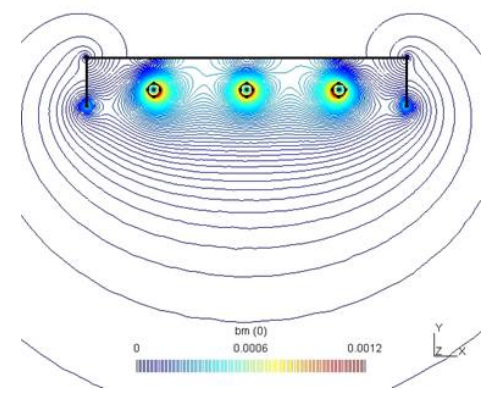

Fig. 13. Field lines in reverse-U shield: a) Aluminum , b) Ferrite.

When influence in RF of geometrical parameters of the shield is analyzed, similar results of those obtained in the horizontal plate case are achieved. For example, Fig. 14 shows the evolution of RF when different values of shield thickness are used. As happened before, RF increases with thickness only in the case of aluminum, establishing at about $10 \mathrm{~mm}$ due to its skin depth, while using steel or ferromagnetic materials the influence is weak.

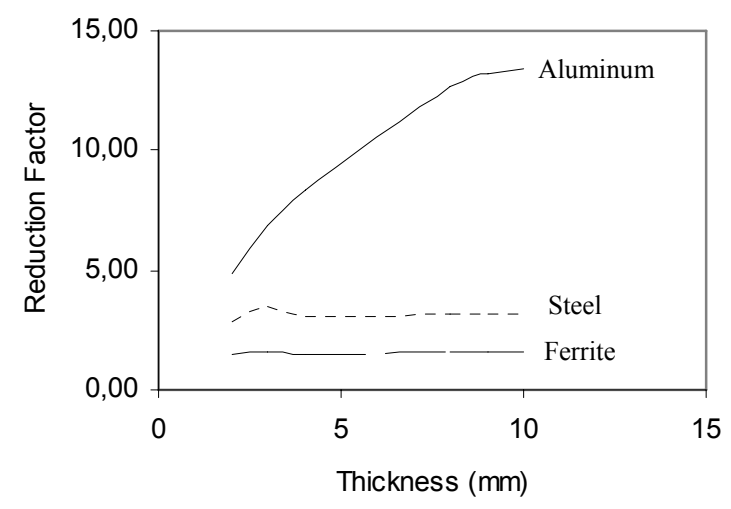

Fig. 14. Evolution of RF with thickness in reverse-U shield.

Another parameter considered is the shield width (Fig. 15), obtaining similar results to the horizontal plate. Aluminum plate seems to be the best solution in general, but in this case better RF is achieved when using a steel shield larger than $2000 \mathrm{~mm}$.

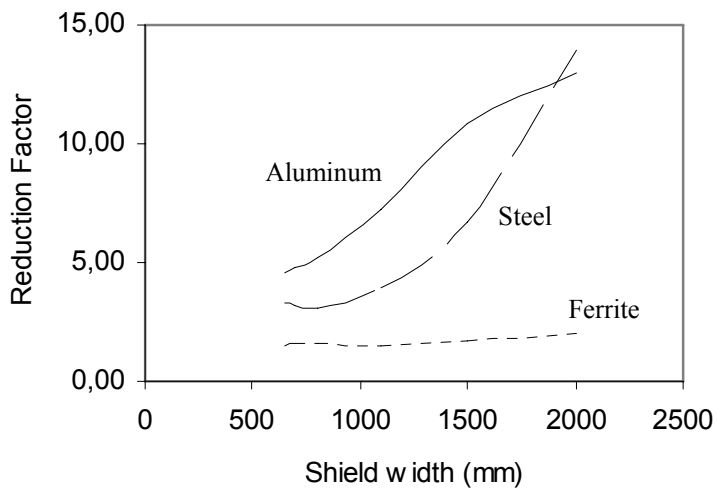

Fig. 15. Evolution of RF with reverse-U shield width.

Studying influence of lateral shield height in the reduction factor, different results are obtained (Fig. 16). In general, $\mathrm{RF}$ increases when lateral plates of the shield get deeper in the soil, but steel has a different behaviour, being the best solution at the value of $400 \mathrm{~mm}$, where it achieves the maximum $\mathrm{RF}$ for all the materials considered.

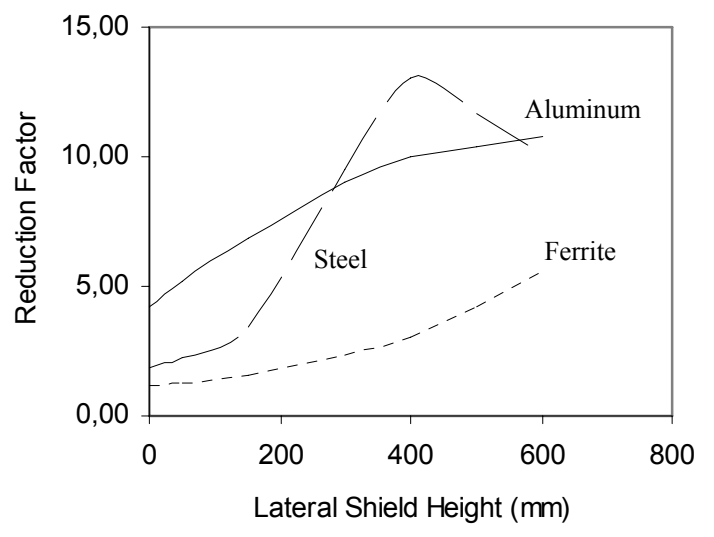

Fig. 16. Evolution of RF with reverse-U shield height.

Another situation to be analyzed is to study the influence on $\mathrm{RF}$ of the mutual distance between the horizontal plate of the shield and the source, when the lateral depth of the shield is fixed (Fig. 17).

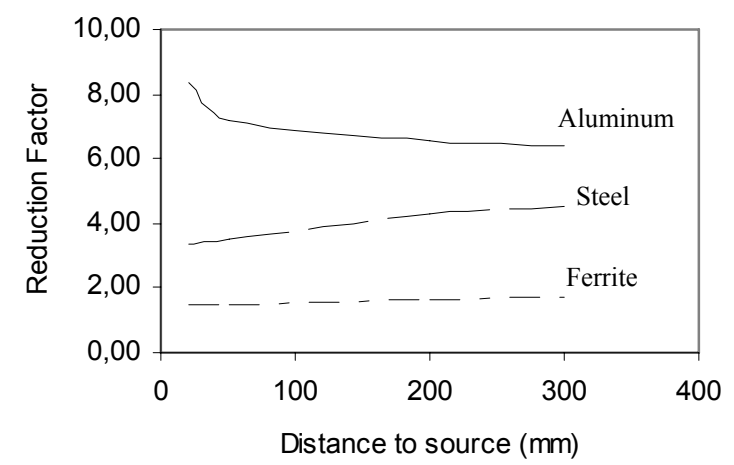

Fig. 17. Evolution of RF with distance between shield and source. 
Different results from the previous case are obtained. In general, influence on RF is quite low. As in the case of a flat shield, RF decreases when aluminium is used, since at larger distance, lower eddy currents are induced. But when ferromagnetic materials are employed, RF increases with the distance. This is possibly because using a higher shape, more flux lines can be diverted into the shield, achieving a better RF performance.

Last parameter considered is the mutual distance between power cables of the line, but this case presents a similar performance than the plate shield case, but getting higher values of RF in the point of interest due to the shield shape.

\section{Closed shield}

The last case studied is considering a closed shield surrounding the cables (Fig. 2b). In this situation ferromagnetic materials have improved its performance, getting results similar of those obtained by aluminum shield, even better (Fig. 18). This solution achieves the best results in the reduction of magnetic field caused by an underground power line.

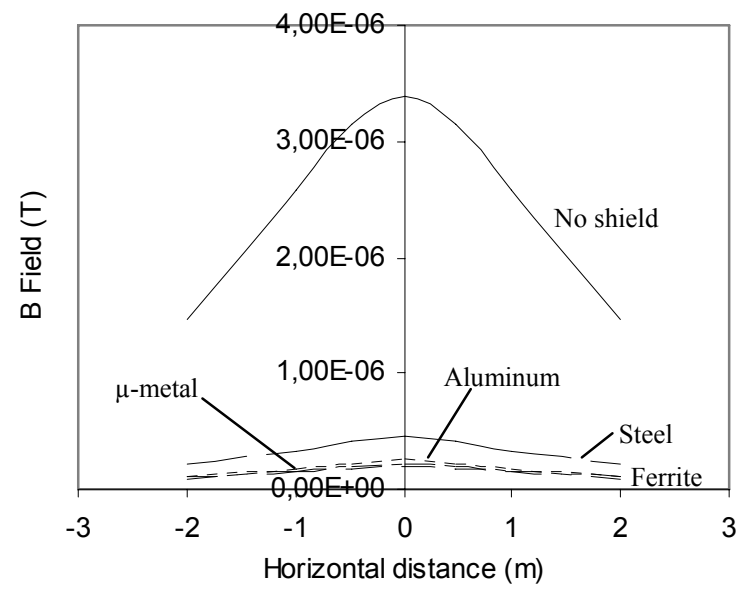

Fig. 18. B field with different materials in a closed shield.

Fig. 19 shows the evolution of RF with the distance from the source. It can be seen how ferromagnetic materials have better RF in the proximities of the source, and even in the area of interest. So this suggests that in the case of using a closed shield, better results will be achieved if ferrite or $\mu$ metal materials are used. This kind of shield, when ferromagnetic materials are used, can divert almost all the field into the shield, getting high values of RF in the outside world of the line (Fig. 20b). On the opposite, steel has the worst reduction, when in previous cases was a reasonable selection.

This solution should be only feasible in new construction underground power lines, since it is complex to implement the whole system in an existing line.

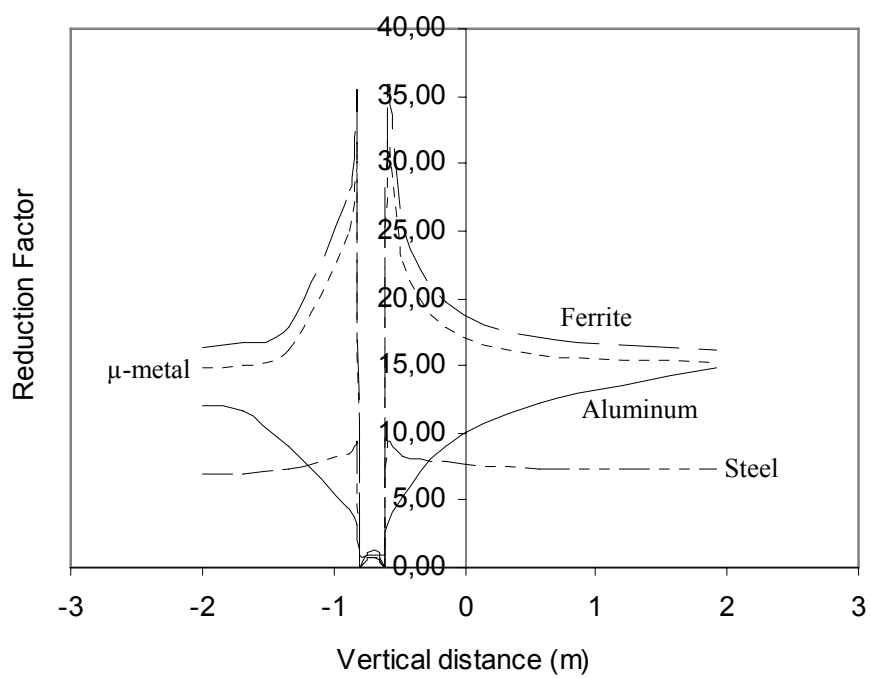

Fig. 19. RF obtained in vertical distance with closed shield.

a)

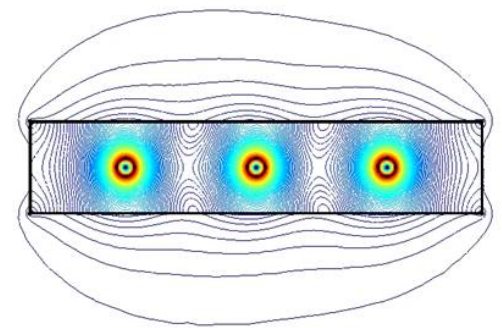

b)

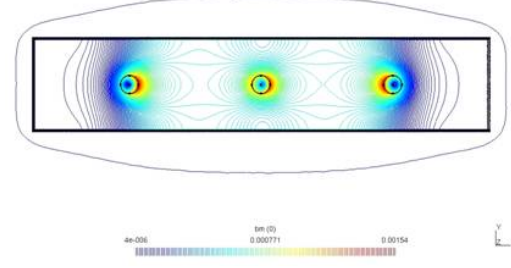

Fig. 20. Shielding method using closed shield.

\section{Conclusion}

The analysis of different shielding configurations has highlighted that passive shielding of magnetic field sources using different plate shapes, strongly depends on the employed material, distance among conductors, situation and dimension of the plate.

The obtained results shows that the use of highly conductive open shields is suitable in case of flat lines, since high field reduction is achieved far from the source (area of interest). In cases where closed shields can be implemented, plates made of ferromagnetic materials are more efficient. Using this kind of materials in open shields has only high field reduction in the proximities of the line. 
Independently of the source configuration, high reduction factor can be obtained by increasing plate thickness when open shield made of high conductive materials are used. In case of ferromagnetic materials, increasing plate thickness has low effect.

Increasing shield width, strongly increases the reduction factor in the point of interest with any of the mentioned materials, but this parameter is limited by the trench width, that has usually a value about $1 \mathrm{~m}$. So, specific site information is required.

Influence of mutual distance among shield and cables have also been done, resulting in an increasing reduction factor when horizontal plates are closer to the field source, whereas in case of reverse-U shields, only aluminum has such trend, while steel or ferrite achieve better performance at higher distance from the source.

In case of reverse- $U$ shield configuration, higher field reduction is obtained when lateral plates of the shield get deeper into the soil. It's been seen that there is a value in which shields made of steel have better performance than using other materials.

It is concluded that, if it is possible to implement (e.g. new underground power lines) better field reduction is achieved when ferromagnetic closed shields are used. On the other hand, in case of mitigating magnetic field on existing lines, the most feasible solution is to install an open shield made of high conductivity materials, such as aluminum or even copper, because of its capacity to withstand corrosion.

Further analysis will concern thermal aspects in these shielding configurations, studying the effect on the ampacity of the power line.

\section{References}

[1] National Radiological Protection Board, "Advice on Limiting Exposure to Electromagnetic Fields $(0-300 \mathrm{GHz})$ ". Doc. NRPB 15 (2) mar. 2004 [Online]. Available:

http://www.hpa.org.uk/radiation/publications/documents_of_ nrpb/abstracts/absd15-2.htm.

[2] A.S. Farag, M.M. Dawoud, I.O. Habiballah, "Implementation of shielding principles for magnetic field management of power cables", Electric Power System Research, 48, pp. 193-209, 1999.

[3] A. Cipollone, A. Fabbri, E. Zendri, "Techniques for shielding underground power lines to minimize the exposure to elf magnetic field in residential areas", International Symposium on Electromagnetic Compatibility, Sorrento, Sept. 2002.

[4] M. D'Amore, E. Menghi, M.S. Sarto, "Shielding techniques of the low-frequency magnetic field from cable power lines", in Proc. 2003 IEEE International Symposium on Electromagnetic Compatibility, vol. 1, pp. 203-08. 